\title{
L'économie et le milieu scientifique bâlois vus par les entreprises de haute technologie et les laboratoires de recherche
}

Economy and scientific environment in point of view of high technology

industries and research groups

Wirtschaft und wissenschaftliche Milieu in der Sicht der

Hochtechnologieunternehmen und Forschungsgruppen

\section{Claudia Saalfrank}

\section{OpenEdition}

Journals

Édition électronique

URL : http://journals.openedition.org/rge/1776

DOI : $10.4000 /$ rge. 1776

ISSN : 2108-6478

Éditeur

Association des géographes de l'Est

Édition imprimée

Date de publication : 1 septembre 2008

ISSN : 0035-3213

\section{Référence électronique}

Claudia Saalfrank, "L'économie et le milieu scientifique bâlois vus par les entreprises de haute technologie et les laboratoires de recherche », Revue Géographique de l'Est [En ligne], vol. 48 / 3-4 I 2008, mis en ligne le 02 mars 2010, consulté le 08 septembre 2020. URL : http://

journals.openedition.org/rge/1776; DOI : https://doi.org/10.4000/rge.1776

Ce document a été généré automatiquement le 8 septembre 2020

Tous droits réservés 


\title{
L'économie et le milieu scientifique bâlois vus par les entreprises de haute technologie et les laboratoires de recherche
}

\author{
Economy and scientific environment in point of view of high technology \\ industries and research groups \\ Wirtschaft und wissenschaftliche Milieu in der Sicht der \\ Hochtechnologieunternehmen und Forschungsgruppen
}

Claudia Saalfrank

\section{Introduction}

1 L'espace économique bâlois connaît depuis 1995 la plus forte croissance régionale de Suisse. Selon le bureau BAK Basel Economics, les taux de croissance annuels ont été de $2,9 \%$ entre 1995 et 2000 et de $2,8 \%$ entre 2004 et 2008 , à comparer avec une croissance moyenne nationale de 2,0\% (Chancellerie d'Etat du canton de Bâle-Ville $2004: 15$ ). En 2003, le canton de Bâle-Ville comptait 9521 entreprises et 178657 emplois, ce qui en fait l'un des plus importants centres économiques de Suisse. Bâle-Ville est le canton suisse qui contribue le plus aux exportations nationales, avec $18,2 \%$ du total de la Suisse, et occupe la seconde place après Zurich en matière d'importations nationales avec 10,3\% (BADAC 2007). La figure 1.1 montre que Bâle constitue également un important site en matière de hautes technologies. Les entreprises de haute technologie et les laboratoires de recherche s'y implantent selon des facteurs de localisation précis, en tenant compte avant tout des propriétés des milieux innovateurs et créatifs qui permettent de créer une forte valeur ajoutée.

2 Dans ce contexte, ce travail se concentre sur les entreprises de haute technologie et les laboratoires de recherche à forte intensité de connaissance, qui constituent les 
« locomotives » du développement économique et de l'innovation au niveau régional et national. Une enquête permet de récolter des données relatives à l'image de Bâle en tant que lieu d'implantation économique et scientifique, à partir desquelles des recommandations concrètes peuvent être formulées en matière de promotion économique et de marketing urbain. L'enquête permet d'évaluer dans quelle mesure ces initiatives sont valables à Bâle-Ville. En outre, le travail permet aux entreprises ou aux laboratoires de recherche d'évaluer l'importance d'un certain nombre de facteurs de localisation pour leurs activités. Les informations récoltées par l'enquête devraient fournir des indications sur l'image de Bâle auprès de ce groupe cible et permettre de déterminer à quel niveau se situent les besoins éventuels.

Figure 1. Nombre d'emplois dans les hautes technologies et quotient de localisation par région MS

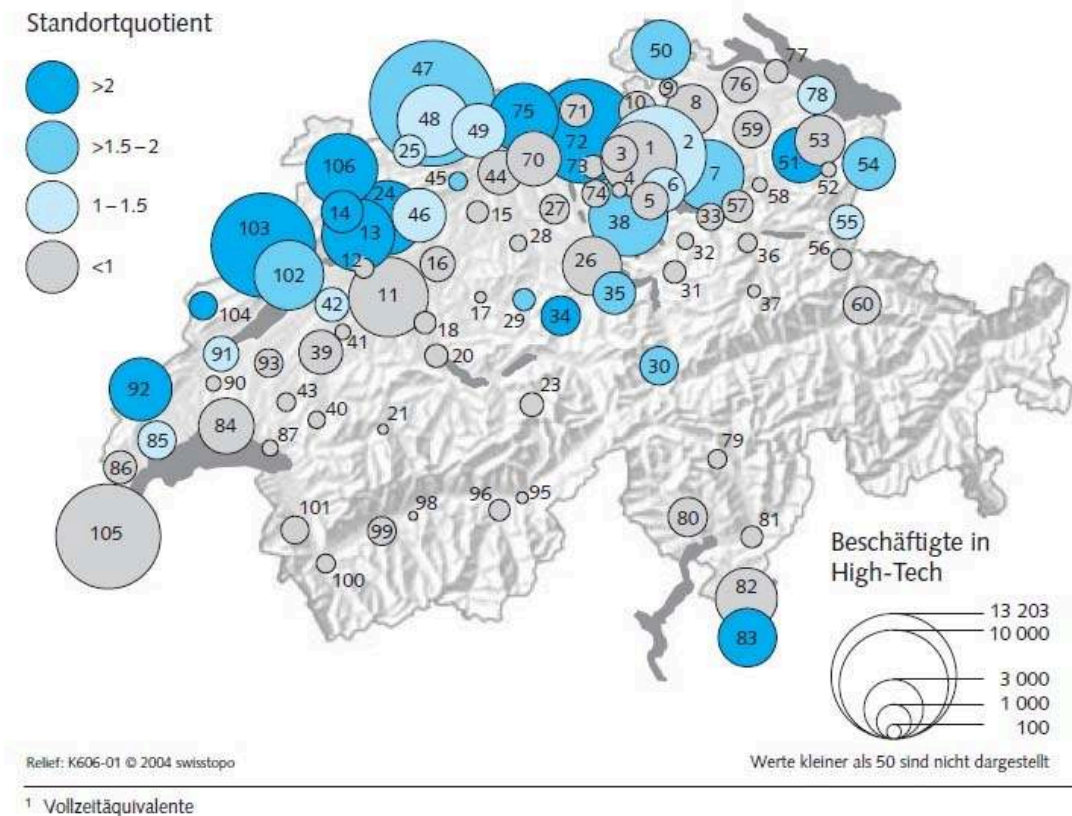

Source : Office fédéral de la statistique 2004 : 30.

La carte (Figure 1) montre le nombre d'emplois dans les secteurs de haute technologie en Suisse (cercles proportionnels) ainsi que le quotient de localisation (couleurs) en 2001. La carte présente les régions MS (Mobilité spatiale) de l'office fédéral de la statistique suisse (OFS), qui correspondent à des niveaux intermédiaires microrégionaux obéissant aux principes des bassins d'emploi. Le quotient de localisation calcule l'importance relative des emplois des secteurs de haute technologie par région MS. Il permet de mettre en évidence le fait que la structure économique de Bâle-Ville est plus fortement orientée vers les hautes technologies que la moyenne suisse et compte le plus grand nombre d'emplois de ce secteur au niveau national (13 203) (Office fédéral de la statistique $2004: 30$ ). 


\section{Données et méthode}

\section{A. Les bases de données utilisées}

4 Dans ce travail, l'accent est mis sur les 700 entreprises et 300 laboratoires de recherche actifs dans le domaine des hautes technologies, dont le siège social principal ou une succursale se trouvent en Suisse et qui peuvent être considérés comme les locomotives du développement régional. La plupart des 700 entreprises concernées ont été sélectionnées en 2001 par l'OFS dans le cadre de son rapport intitulé Standorte der innovativen Schweiz (Lieux d'implantation d'activités innovantes en Suisse). Les secteurs de haute technologie y ont été sélectionnés sur la base d'une classification de l'OCDE selon leurs codes NOGA (Nomenclature générale des activités économiques) (Office fédéral de la statistique $2004: 27$ ). En Suisse, les entreprises sont considérées comme marquées par une haute intensité de Recherche \& Développement (R\&D), si elles investissent au moins $8 \%$ de leur chiffre d'affaires dans cette activité. La nomenclature NOGA est un instrument de travail fondamental qui permet de structurer, d'analyser et de représenter l'information statistique. Elle permet de classer les entreprises et les emplois selon leur activité économique et de présenter les catégories obtenues de manière claire et homogène (Office fédéral de la statistique 2006:3). Le rapport de l'OFS précise que la classification de l'OCDE a été modifiée de manière minime en ce qui concerne le secteur «Fabrication d'appareils électriques pour la production, la distribution de l'électricité et d'autres activités similaires " (NOGA 31). Ceci est justifié, d'une part, par le fait que ce secteur comporte le taux le plus important d'intensité en R\&D dans la catégorie des moyennes-hautes technologies et, d'autre part, parce que ce secteur est très fortement basé sur les technologies en Suisse.

5 Compte tenu de cette modification, les secteurs définis par l'OCDE sont indiqués ciaprès par leurs codes NOGA respectifs :

- Fabrication de produits pharmaceutiques (NOGA 24.4)

- Fabrication de machines de bureau, d'appareils pour le traitement des données et d'équipements informatiques (NOGA 30)

- Fabrication d'appareils électriques pour la production, la distribution de l'électricité et d'autres activités similaires (NOGA 31)

- Fabrication d'équipements de radio, de télévision et de communication (NOGA 32)

- Fabrication d'instruments médicaux et d'instruments de précision et d'optique; horlogerie (NOGA 33)

- Construction aéronautique et spatiale (NOGA 35.3)

6 L'enquête a concerné les membres de l'Association des sociétés pharmaceutiques suisses pratiquant la recherche Interpharma et une partie des membres de la SGCI Chemie Pharma Schweiz, de manière à couvrir l'industrie pharmaceutique. Les membres d'Interpharma sont des entreprises pharmaceutiques engagées dans la recherche et qui produisent et commercialisent au moins un médicament sur le marché national. La SGCI Chemie Pharma Schweiz est l'organisation faîtière de l'industrie chimique et pharmaceutique suisse. Ses quelque 200 sociétés membres sont principalement actives dans la recherche, le développement, la fabrication ou la vente de spécialités pharmaceutiques, de vitamines, de produits chimiques spécialisés, de produits phytosanitaires, ainsi que d'arômes et de parfums. Les coordonnées des entreprises actives dans le domaine de la fabrication de machines de bureau et 
d'équipements informatiques, d'appareils électriques pour la production, la distribution de l'électricité, et d'équipements de radio, de télévision et de communication proviennent de plusieurs sources. Une grande partie de ces adresses a été fournie par Electrosuisse, la principale association suisse du domaine de l'électrotechnique tandis que d'autres proviennent de bases de données traditionnelles. Le secteur de la fabrication d'instruments médicaux, d'instruments de précision et d'optique ou l'horlogerie correspond aux membres de la Fédération des associations suisses du commerce et de l'industrie de la technologie médicale (Fasmed). Les entreprises actives dans le secteur de la construction aéronautique et spatiale ont été sélectionnées sur la base de banques de données d'adresses conventionnelles.

7 Afin de prendre en considération certaines entreprises qui ne figurent pas dans la classification de l'OCDE mais qui pratiquent des activités innovantes et à forte intensité de connaissance, l'enquête a également porté sur les secteurs suivants: industrie chimique, biotechnologies, technologies de l'information et de la communication (TIC); micro et nanotechnologies, techniques de l'environnement. Dans l'industrie chimique, les entreprises membres de la SGCI Chemie Pharma Schweiz ont été sélectionnées alors que dans les biotechnologies, ce sont les membres de l'Association suisse de biotechnologie (Swiss Biotech Association) qui ont été sélectionnés en raison de leurs activités dans le domaine de la génétique et de l'intensité de leur R\&D. L'enquête a pris en considération les entreprises actives dans le champ des technologies de l'information et de la communication (TIC), pour lesquelles la Suisse est renommée. Les entreprises suisses constituent en effet des pionniers dans le domaine et jouent un rôle majeur dans ce genre de technologies (Location Switzerland 2007a). Les dépenses par habitant de la Suisse sont d'ailleurs les plus élevées de tous les pays de l'OCDE en 2001 (Office fédéral de la statistique 2003: 3). Dans ce domaine, les entreprises ont été sélectionnées sur la base des membres de SwissICT, l'Association suisse des technologies de l'information et de la communication et à partir de listes d'adresses traditionnelles.

8 Les micro- et nanotechnologies sont l'un des secteurs possédant le plus grand potentiel d'innovation. La Suisse dispose dans ce domaine d'une connaissance scientifique et technologique avancée. Les entreprises du secteur ont été sélectionnées sur la base des membres de l'Association pour l'application et la promotion des technologies électroniques (APTE). Les entreprises actives dans le secteur des techniques de l'environnement ont été sélectionnées à partir des membres de l'Association suisse pour les techniques de l'environnement (SVUT). La Suisse occupe une position de premier plan dans le domaine de la défense de l'environnement et du recyclage et son cluster basé sur les technologies environnementales constitue un important exportateur de services et de produits liés au développement durable (Location Switzerland 2007b). En outre, la Suisse est fortement représentée dans le domaine des brevets relatifs aux techniques environnementales à l'échelle mondiale (office fédéral de la statistique $1999: 2$ ).

En ce qui concerne les laboratoires de recherche, une base de données de 800 équipes comprenant l'ensemble des universités et des hautes écoles suisses a été utilisé. A partir de cette base de données, un échantillon comprenant 200 laboratoires de recherche travaillant dans les universités et les écoles polytechniques suisses de Zurich et de Lausanne a été contacté par courrier. 


\section{B. Choix des facteurs de localisation}

10 Outre des informations spécifiques à chaque entreprise ou laboratoires de recherche telles que le secteur, la localisation et le siège social, l'enquête s'est focalisée sur les facteurs qui, au vu des entreprises et des laboratoires de recherche, devaient faire l'objet d'une évaluation dans le canton de Bâle-Ville. Sur la base de la littérature existante dans ce domaine et d'analyses relatives aux représentations, les dimensions suivantes ont été identifiées et insérées au questionnaire (Figure 2).

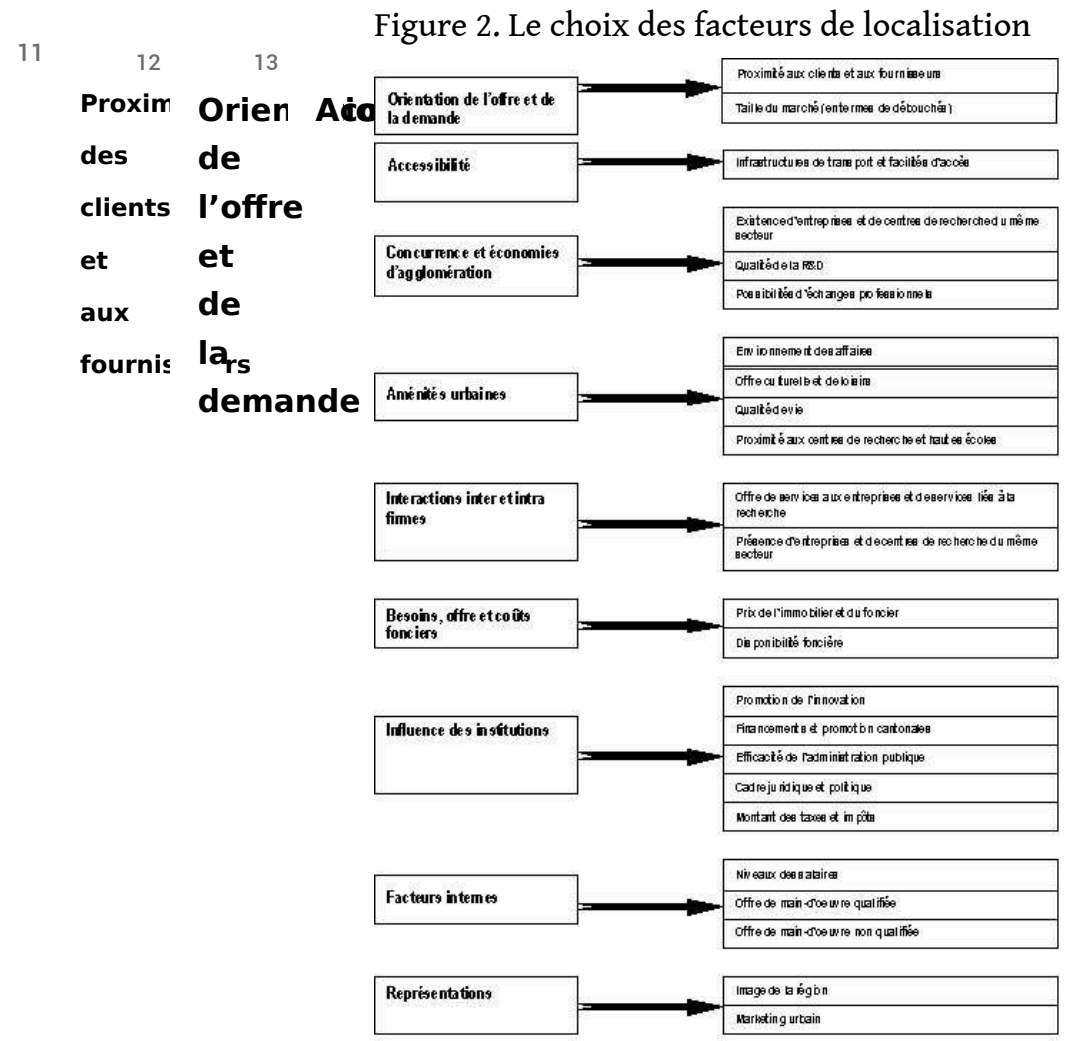

Source : Heineberg et DeLange (2006 : 183), modifié par l'auteur.

14 Les facteurs de localisation reconnus comme importants dans l'enquête ont été identifiés à partir des analyses de Heineberg et DeLange (2006). Ces auteurs ont développé un ensemble de conditions et de facteurs relatifs aux activités des secteurs tertiaires et quaternaires.

\section{Bâle comme lieu d'implantation des activités économiques et scientifiques}

\section{A. La perception de Bâle parmi les entreprises de haute technologie et les laboratoires de recherche}

Il ressort de l'évaluation globale que les personnes interrogées perçoivent Bâle comme une ville indiscutablement internationale. Ce sentiment est lié à la forte concentration d'entreprises multinationales actives dans le domaine de la chimie et de la pharmacie, à la présence de nombreux spécialistes internationaux ainsi qu'aux manifestations 
d'envergure internationale comme ArtBasel ou BaselWorld auxquelles s'ajoutent de nombreuses foires et congrès. Les personnes interrogées relèvent également la présence de l'EuroAirport Bâle-Mulhouse-Fribourg, des gares de la SNCF et de la Deutsche Bahn, qui se trouvent à proximité de la ville, et du port du Rhin qui constitue la seule infrastructure portuaire de fret de Suisse, permettant de relier le pays à l'océan Atlantique. En outre, on peut supposer que la situation de Bâle à la frontière entre Suisse, France et Allemagne contribue au caractère international de la ville. Les personnes interrogées notent que les qualificatifs " accueillante pour les entreprises", "accueillante pour les investisseurs", "accueillante pour les technologies", «innovante» et "moderne" correspondent bien à la ville de Bâle. Bien qu'ils apparaissent un peu moins nettement que le qualificatif «internationale», ces attributs peuvent être généralement considérés comme positifs. En revanche, les personnes interrogées notent que les qualificatifs «dynamique ", «accueillante pour les familles » et « centrale » ne correspondent pas à l'image de Bâle.

\section{B. Les facteurs attractifs et répulsifs d'une localisation à Bâle.}

Les dix facteurs les plus fréquemment cités en faveur d'une localisation à Bâle sont les suivants :

- La forte concentration d'entreprises chimiques et pharmaceutiques

- La situation aux frontières de trois Etats

- La bonne accessibilité (navigation sur le Rhin, voies de circulation, aéroport)

- Le caractère international de la ville

- La qualité de l'offre de main-d'œuvre qualifiée

- L'image positive de la ville

- L'EuroAirport Bâle-Mulhouse-Fribourg

- Le bon état général des infrastructures

- L'Université (réputation et haut potentiel)

- La situation centrale en Europe

17 L'enquête montre que les dix facteurs les plus répulsifs liés à une localisation à Bâle sont les suivants :

- Situation excentrée par rapport à la Suisse

- Forte imposition fiscale

- Coût de la vie élevé

- Disponibilité limitée des surfaces constructibles

- Environnement politique (gauche et écologistes)

- Congestion

- Liaisons de transports défavorables

- Non adhésion de la Suisse à l'UE

- Caractère insuffisamment international de l'EuroAirport

- Lourdeur des processus de prise de décision dans l'administration 


\section{La pertinence de certains facteurs de localisation dans la décision d'implantation des entreprises et des laboratoires de recherche}

Afin de déterminer la pertinence des facteurs de localisation qui pourraient s'appliquer aux entreprises de haute technologie et aux laboratoires de recherche, une liste de facteurs, dont l'importance est graduée de «très important » à " pas important », a été proposée.

Tableau 2.1. Pertinence des facteurs de localisation selon les personnes interrogées

\begin{tabular}{|c|c|c|c|c|c|}
\hline $\begin{array}{l}\text { Facteurs de } \\
\text { localisation }\end{array}$ & $\begin{array}{l}\text { Très } \\
\text { important, } \\
\text { en \% }\end{array}$ & $\begin{array}{l}\text { Important, } \\
\text { en \% }\end{array}$ & $\begin{array}{l}\text { Peu } \\
\text { important, } \\
\text { en\% }\end{array}$ & $\begin{array}{l}\text { Pas } \\
\text { important, } \\
\text { en \% }\end{array}$ & $\begin{array}{l}\text { Total, } \\
\text { en } \%\end{array}$ \\
\hline $\begin{array}{l}\text { Orientation } \\
\text { l'offre et de la } \\
\text { demande }\end{array}$ & & & & & \\
\hline $\begin{array}{l}\text { Proximité des clients } \\
\text { et des fournisseurs }\end{array}$ & 29,1 & 30,0 & 30,0 & 10,9 & 100,0 \\
\hline $\begin{array}{l}\text { Taille du marché (en } \\
\text { termes de débouchés) }\end{array}$ & 24,6 & 32,7 & 18,2 & 24,5 & 100,0 \\
\hline \multicolumn{6}{|l|}{ Accessibilité } \\
\hline $\begin{array}{l}\text { Infrastructures de } \\
\text { transport et facilités } \\
\text { d'accès }\end{array}$ & 29,1 & 44,5 & 19,1 & 7,3 & 100,0 \\
\hline \multicolumn{6}{|l|}{$\begin{array}{l}\text { Concurrence } \\
\text { économies } \\
\text { d'agglomération }\end{array}$} \\
\hline $\begin{array}{l}\text { Existence } \\
\text { d'entreprises et de } \\
\text { centres de recherche } \\
\text { du même secteur }\end{array}$ & 25,5 & 40,0 & 21,8 & 12,7 & 100,0 \\
\hline Qualité de la R\&D & 59,2 & 22,7 & 14,5 & 3,6 & 100,0 \\
\hline $\begin{array}{l}\text { Possibilités } \\
\text { d'échanges } \\
\text { professionnels }\end{array}$ & 20,9 & 40,9 & 30,0 & 8,2 & 100,0 \\
\hline \multicolumn{6}{|l|}{ Aménités urbaines } \\
\hline $\begin{array}{l}\text { Environnement des } \\
\text { affaires }\end{array}$ & 30,0 & 55,5 & 10,9 & 3,6 & 100,0 \\
\hline
\end{tabular}




\begin{tabular}{|c|c|c|c|c|c|}
\hline $\begin{array}{l}\text { Offre culturelle et de } \\
\text { loisirs }\end{array}$ & 11,0 & 51,8 & 24,5 & 12,7 & 100,0 \\
\hline Qualité de vie & 17,3 & 59,1 & 17,3 & 6,3 & 100,0 \\
\hline $\begin{array}{l}\text { Proximité des centres } \\
\text { de recherche et } \\
\text { grandes écoles }\end{array}$ & 38,2 & 38,2 & 20,9 & 2,7 & 100,0 \\
\hline $\begin{array}{l}\text { Interactions inter et } \\
\text { intra firmes }\end{array}$ & & & & & \\
\hline $\begin{array}{l}\text { Offre de services aux } \\
\text { entreprises et de } \\
\text { services liés à la } \\
\text { recherche }\end{array}$ & 17,3 & 53,6 & 20,9 & 8,2 & 100,0 \\
\hline $\begin{array}{l}\text { Présence } \\
\text { d'entreprises et de } \\
\text { centres de recherche } \\
\text { du même secteur }\end{array}$ & 25,5 & 40,0 & 21,8 & 12,7 & 100,0 \\
\hline $\begin{array}{l}\text { Besoins, offre et } \\
\text { coûts fonciers }\end{array}$ & & & & & \\
\hline $\begin{array}{l}\text { Prix de l'immobilier } \\
\text { et du foncier }\end{array}$ & 5,5 & 45,5 & 35,5 & 13,5 & 100,0 \\
\hline Disponibilité foncière & 7,3 & 39,1 & 33,6 & 20,0 & 100,0 \\
\hline $\begin{array}{l}\text { Influence des } \\
\text { institutions }\end{array}$ & & & & & \\
\hline $\begin{array}{l}\text { Promotion } \quad \text { de } \\
\text { l'innovation }\end{array}$ & 29,2 & 44,5 & 23,6 & 2,7 & 100,0 \\
\hline $\begin{array}{l}\text { Financement et } \\
\text { promotion cantonales }\end{array}$ & 19,1 & 30,0 & 35,5 & 15,4 & 100,0 \\
\hline $\begin{array}{l}\text { Efficacité } \quad \text { de } \\
\text { l'administration } \\
\text { publique }\end{array}$ & 31,0 & 40,0 & 24,5 & 4,5 & 100,0 \\
\hline $\begin{array}{l}\text { Cadre juridique et } \\
\text { politique }\end{array}$ & 24,5 & 48,2 & 20,9 & 6,4 & 100,0 \\
\hline $\begin{array}{l}\text { Montant des taxes et } \\
\text { impôts }\end{array}$ & 24,5 & 48,2 & 20,0 & 7,3 & 100,0 \\
\hline Facteurs internes & & & & & \\
\hline
\end{tabular}




\begin{tabular}{|l|l|l|l|l|l|}
\hline Niveau des salaires & 5,4 & 19,1 & 56,4 & 19,1 & 100,0 \\
\hline $\begin{array}{l}\text { Offre de main- } \\
\text { d'œuvre qualifiée }\end{array}$ & 67,3 & 23,6 & 6,4 & 2,7 & 100,0 \\
\hline $\begin{array}{l}\text { Offre de main- } \\
\text { d'œuvre non qualifiée }\end{array}$ & 1,8 & 10,9 & 31,8 & 55,5 & 100,0 \\
\hline Représentations & & & & & \\
\hline Image de la région & 8,2 & 52,7 & 30,0 & 9,1 & 100,0 \\
\hline Marketing urbain & 4,5 & 12,7 & 39,1 & 43,7 & 100,0 \\
\hline
\end{tabular}

Source: l'auteur, enquête 2006

19 Les facteurs de localisation jugés « très importants » par les personnes interrogées pour leurs entreprises ou laboratoires de recherche sont : l'offre de main-d'œuvre qualifiée $(67,3 \%)$, la qualité de la $R \& D(59,2 \%)$ et la proximité aux centres de recherche et aux hautes écoles $(38,2 \%)$. Les facteurs jugés "pas importants» par les personnes interrogées sont l'offre de main-d'œuvre non qualifiée (55,5\%) et le marketing urbain tel qu'il était pratiqué en 2007 (43,7\%). A noter que le marketing urbain et l'administration ont connu de profondes transformations liées à la réforme administrative cantonale depuis cette enquête.

Compte tenu de la nature des entreprises et des laboratoires concernés, ces résultats ne sont guère surprenants. En effet, les investissements en R\&D consentis par ces derniers sont très dépendants de la proximité des centres de recherche et des grandes écoles, et de l'environnement qualitatif dans lequel s'effectue la R\&D. En outre, le fait de disposer d'une main-d'œuvre qualifiée constitue une condition indispensable pour l'activité de ces entreprises actives dans le développement de nouvelles technologies ou dans le milieu de la recherche. Cela explique, en parallèle, que la disponibilité en main-d'œuvre non qualifiée ne constitue pas un facteur de localisation important. Il est en revanche plus difficile d'expliquer pourquoi les personnes interrogées attribuent si peu d'importance au marketing urbain. Peut-être ces personnes ne disposent-elles que de trop peu d'informations relatives au marketing urbain et sont-elles enclines à penser que ce marketing n'est pas utile pour leurs activités.

\section{Evaluation de certains facteurs de localisation relatifs à Bâle-Ville}

21 Les éléments qui suivent décrivent l'image véhiculée par Bâle-Ville auprès des entreprises de haute technologie et des laboratoires de recherche. Les facteurs identifiés sont identiques à ceux qui ont été précédemment décrits au chapitre 2.3. Ils permettent de mettre en évidence les principales caractéristiques de Bâle-Ville.

Orientation de l'offre et de la demande. Les facteurs de localisation relatifs à la taille du marché et à la proximité des clients et des fournisseurs permettent de faire ressortir les perceptions spécifiques au marché local. Les chiffres montrent que $46 \%$ des personnes interrogées considèrent la taille du marché bâlois en termes de débouchés comme « très bonne » ou « bonne » tandis qu'un cinquième d'entre-elles la considèrent 
comme " mauvaise " ou "très mauvaise ". Une proportion importante des personnes interrogées ne se prononce pas sur la question (34\%). La proximité aux clients et aux fournisseurs est considérée par $66 \%$ des personnes interrogées comme "très bonne » ou « bonne », tandis que $20 \%$ des personnes ne peuvent se prononcer sur la question.

Accessibilité. Bâle est clairement considérée par les entreprises et les laboratoires de recherche comme une ville bénéficiant de bonnes infrastructures et d'un accès facile : $34 \%$ des personnes interrogées jugent ce critère « très bon » et $50 \%$ «bon ", tandis que $8 \%$ jugent l'accessibilité « mauvaise » ou ne peuvent se prononcer. La situation de Bâle comme "Porte dorée» de la Suisse s'est dégradée depuis quelques années, consécutivement à l'évolution générale de l'économie mondiale et sa dématérialisation (Frey 1989 : 11). Cependant, la ville demeure un carrefour d'importance continentale en matière de transports: située au bord du Rhin, Bâle-Ville est ainsi située sur la voie fluviale la plus importante d'Europe, bénéficie de la proximité à l'aéroport trinational de Bâle-Mulhouse-Fribourg, possède un important nœud ferroviaire doté de l'une des plus grandes gares de triage d'Europe (Muttenz) et se situe au carrefour de plusieurs autoroutes européennes, notamment celles qui permettent de traverser les Alpes vers le sud de l'Europe. Le fait que Bâle dispose de bonnes connexions fluviales et ferroviaires renforce son importance dans le contexte de l'intégration européenne et de la protection de l'environnement. Ces éléments confirment que Bâle-Ville peut être considéré comme un canton possédant de bonnes infrastructures et une bonne accessibilité.

24 Concurrence et économies d'agglomération. Il ressort de l'enquête que $78 \%$ des personnes interrogées jugent les possibilités d'échanges professionnels «très bonnes » ou «bonnes» tandis que la qualité de la R\&D est appréciée par $81 \%$ des personnes enquêtées selon les mêmes critères. L'existence d'entreprises ou de centres de recherche travaillant dans le même secteur est considérée par $75 \%$ des personnes interrogées comme "très bonne " ou "bonne ». A noter que de très rares personnes jugent ces facteurs « mauvais » ou « très mauvais » à Bâle-Ville. A elles seules, les foires organisées par MCH Messe Schweiz SA attirent 7998 exposants et près d'un million de visiteurs chaque année à Bâle (Basel Economics $2006: 4$ ), ce qui représente 22 exposants et 2700 visiteurs par jour. Ces chiffres montrent clairement dans quelle mesure les foires organisées à Bâle jouent un rôle important dans les échanges entre professionnels et contribuent à créer ou à consolider les relations internationales. Dans le cadre du Recensement des entreprises 2005 organisé par l'OFS, l'évolution de la R\&D est décrite de la manière suivante: «La hausse des services aux entreprises a été quelque peu freinée au cours de ces dernières années, à l'exception de la R\&D, dont le nombre de salariés a quasiment doublé depuis 2001 » (Office statistique du canton de Bâle-Ville $2006: 2$, notre traduction). Bien que ces données quantitatives ne fournissent pas d'informations sur la qualité des emplois créés, cette augmentation est sans doute liée à une hausse de la demande et à une amélioration de la qualité fournie par les entreprises. Dans son Rapport sur la politique économique 2003, le canton de Bâle-Ville évoque le fait que les dépenses de la région en matière de recherche fondamentale et de recherche appliquée ont fortement augmenté. A cela s'ajoute le fait que de nombreux centres de recherche privés se sont implantés à Bâle-Ville (Département de l'économie et des affaires sociales du canton de Bâle-Ville 2003: 42) et qu'il existe une forte concentration de chercheurs dans le domaine des sciences de la vie. Une telle 
concentration d'activités est susceptible d'intensifier et d'accélérer les processus d'innovation.

Besoins, offre et coûts fonciers. Bâle-Ville est soumise à un manque chronique de surfaces constructibles. Il n'est presque plus possible d'y trouver des espaces disponibles pour de nouvelles constructions, alors que les surfaces de bureaux sont encore suffisants. Le manque de surfaces disponibles, lié à la prospérité bâloise, alimente la hausse des rendements et des coûts fonciers, ce qui a un effet sur les loyers (Office statistique du canton de Bâle-Ville 1999: 35). Les entreprises désireuses de s'agrandir ou de construire de nouvelles installations sont confrontées à des prix élevés ou à une absence de disponibilité foncière, ce qui les pousse à se localiser ailleurs qu'à Bâle-Ville.

Influence des institutions. Les mesures concrètes prises en faveur de l'innovation concernent le cofinancement de BioValley, l'augmentation du budget de la promotion économique, des initiatives destinées à communiquer sur les points forts de la ville ainsi que des facilités liées au parc biotechnologique cantonal (Département de l'économie et des affaires sociales du canton de Bâle-Ville $2003: 44$ ).

Représentations. L'image de Bâle-Ville est jugée positivement par les personnes interrogées, qui sont $14 \%$ à l'évaluer "très bonne ", $60 \%$ "bonne » et seulement $11 \%$ "mauvaise ", tandis que $15 \%$ n'ont pas d'opinion à ce sujet. Le marketing urbain de Bâle-Ville est jugé «très bon " ou «bon " par $41 \%$ des personnes enquêtées, alors que près de la moitié des personnes interrogées $(45 \%)$ n'ont aucune opinion à ce sujet. Ce résultat surprenant permet de conclure que le marketing urbain tel qu'il a été conçu depuis 2000 demeure peu connu.

Conclusion intermédiaire. Douze des 23 facteurs de localisation obtiennent des appréciations «très bonnes » ou "bonnes" aux yeux des personnes interrogées. Il s'agit de :

- L'offre de main-d'œuvre qualifiée (75\%)

- Les infrastructures de transport et facilités d'accès (84\%)

- L'offre de services aux entreprises et de services liés à la recherche (59\%)

- La proximité des clients et des fournisseurs (66\%)

- La proximité des centres de recherche et des grandes écoles (79\%)

- L'existence d'entreprises et de centres de recherche du même secteur (75\%)

- La possibilité d'échanges professionnels (78\%)

- La qualité de vie $(68 \%)$

- L'offre de culture et de loisirs (78\%)

- La qualité de la R\&D (81\%)

- L'image de la région $(74 \%)$

- L'environnement des affaires (69\%)

Les facteurs de localisation suivants ont reçu la plus forte proportion d'appréciations «très bonnes » :

- La qualité de la R\&D (39\%)

- La proximité des centres de recherche et des grandes écoles (39\%)

- L'existence d'entreprises et de centres de recherche du même secteur (37\%)

- Les infrastructures de transport et facilités d'accès (34\%) 

mauvais » par les personnes interrogées :

- Le niveau des salaires (35\%)

- Les prix de l'immobilier et du foncier (52\%)

- Le montant des taxes et impôts (43\%)

- La disponibilité des surfaces constructibles (42\%)

31 Les résultats de cette enquête montrent que les entreprises de haute technologie et les laboratoires de recherche ont une perception de Bâle qui correspond en règle générale aux caractéristiques objectives du lieu. Il est cependant frappant de constater la forte proportion de personnes interrogées qui déclarent n'avoir aucune opinion sur les questions posées, notamment sur les facteurs relatifs aux niveaux des salaires, à la disponibilité en surfaces constructibles, à la promotion de l'innovation, aux financements cantonaux, au marketing urbain et à l'efficacité de l'administration publique. Cela pourrait signifier que les entreprises interrogées n'ont encore jamais débattu des principaux facteurs de localisation qui concernent Bâle-Ville. Les entreprises qui n'ont jamais eu l'intention de construire des bureaux à Bâle, par exemple, ne peuvent s'exprimer sur la disponibilité des surfaces constructibles ou sur les prix immobiliers et fonciers. Il est également possible que les personnes sans avis ne possèdent que trop peu d'informations sur les facteurs de localisation du canton. L'enquête permet de mettre en évidence que les entreprises et les laboratoires de recherche ont potentiellement besoin de mieux connaître les avantages de Bâle-Ville du point de vue de la localisation.

En résumé, très peu de personnes interrogées ont une mauvaise image de Bâle-Ville. Ces personnes ont alors soit une bonne image de la ville, soit ils n'en possèdent pas du tout. En outre, on peut retenir que les facteurs de localisation sont appréhendés d'une manière bien particulière. Peu de gens notent en effet qu'un facteur de localisation est particulièrement "bon" ou "très bon » pour leurs entreprises ou laboratoires et jugent que tous les autres sont "mauvais » ou «très mauvais ». La grande majorité notent plutôt qu'un facteur est «bon » ou « très bon » et concluent ensuite qu'ils n'ont pas d'opinion sur les autres. Les plus grandes différences entre les facteurs biens ou très bien notés et les facteurs mal ou très mal notés concernent les niveaux des salaires, les niveaux des taxes et impôts et la taille du marché en termes de débouchés.

Une comparaison des perceptions des entreprises de haute technologie et des laboratoires de recherche, ainsi que des caractéristiques de Bâle-Ville peut être réalisée de deux manières différentes. Il est tout d'abord possible de comparer les facteurs de localisation jugés « importants » ou « très importants » avec les caractéristiques de Bâle jugées « bonnes » ou « très bonnes » et de trouver une éventuelle correspondance entre les deux. Trois facteurs correspondent à cette situation: l'offre de main-d'œuvre qualifiée, la proximité des centres de recherche et des grandes écoles, et la qualité de la R\&D. En comparant ensuite les facteurs de localisation considérés comme très importants et les caractéristiques de Bâle-Ville jugées "très bonnes ", le résultat n'est pas concordant. Des divergences partielles sont observées entre les besoins et la perception des lieux : de graves anomalies sont observées en particulier dans six des 23 facteurs de localisation et de faibles écarts dans la quasi-totalité des autres. Les plus grands écarts concernent la qualité de la R\&D, l'environnement des affaires, la promotion de l'innovation, l'efficacité de l'administration publique, le niveau des taxes et impôts ainsi que l'offre de main-d'œuvre qualifiée. 


\section{E. Les implications pour l'avenir international de Bâle-Ville}

$\mathrm{du}$ positionnement international de Bâle-Ville. L'enquête montre que $21 \%$ des personnes interrogées sont optimistes sur l'avenir du développement économique et scientifique du canton à l'échelle internationale, qui pourrait alors éventuellement conduire à une hausse de la croissance économique, à une hausse du nombre d'entreprises et de laboratoires de recherche qui s'établiraient dans le canton et à une plus grande visibilité de Bâle-Ville vis-à-vis de l'extérieur. Les personnes enquêtées sont $23 \%$ à juger que l'évolution prise par le canton en matière de développement économique est négative et déclinante. Selon eux, cette évolution pourrait conduire à une baisse de la croissance économique et à une perte d'importance de Bâle-Ville, synonyme de fermetures d'entreprises. Le reste des personnes interrogées, soit 56\%, pense que le poids international et la notoriété de Bâle resteront inchangés et imagine que les créations d'entreprises et de centres dédiés à la recherche ainsi que la croissance économique seront modérés.

\section{Conclusion}

Les résultats présentés dans ce travail montrent que l'image de Bâle-Ville demeure globalement positive. Il faut cependant noter qu'une proportion importante des personnes enquêtées ne se prononce pas sur l'importance des facteurs de localisation soumis à l'analyse. Les proportions les plus élevées de personnes n'ayant aucun avis se rapportent aux niveaux des salaires, à la promotion de l'innovation, aux financements cantonaux en matière de recherche, au marketing urbain et à l'efficacité de l'administration publique. Les facteurs de localisations jugés les plus caractéristiques et les plus importants par les personnes enquêtées sont l'offre de main-d'œuvre qualifiée, la qualité de la R\&D et la proximité des centres de recherche et des grandes écoles. Ces personnes relèvent que Bâle-Ville possède un potentiel particulièrement élevé dans plusieurs domaines: qualité de la R\&D, proximité des centres de recherche et des grandes écoles, existence d'entreprises et de laboratoires du même secteur, infrastructures de transport et facilités d'accès. Les déficits les plus importants relevés concernent le niveau des salaires, le coût de l'immobilier et du foncier, le niveau des taxes et impôts ainsi que la disponibilité des surfaces constructibles.

Une question ouverte a permis de montrer que les trois raisons principales qui faisaient de Bâle-Ville une localisation intéressante en matière économique et scientifique étaient les suivantes: forte concentration des entreprises chimiques et pharmaceutiques, situation avantageuse à la frontière de trois Etats, facilité d'accès. A l'opposé, les trois raisons les plus désavantageuses d'une localisation à Bâle-Ville sont, de l'avis des personnes enquêtées : la situation excentrée de la ville par rapport au reste de la Suisse, l'imposition fiscale et le niveau élevé des prix. La majorité des personnes interrogées (56\%) estime que le rôle international de Bâle-Ville restera inchangé dans le futur, tandis que $21 \%$ sont optimistes sur l'avenir de la ville et $23 \%$ pessimistes.

Recommandations. Les résultats obtenus montrent que certains facteurs de localisation avantageux pour Bâle-Ville ne sont pas perçus par les entreprises et les laboratoires de recherche. En parallèle des politiques menées pour favoriser

Revue Géographique de l'Est, vol. 48 / 3-4 | 2008 
l'implantation des entreprises, un soin particulier devrait être apporté aux entreprises et laboratoires déjà présents dans la région. Le marketing urbain pourrait jouer un rôle important dans cette démarche et devrait par conséquent voir ses compétences renforcées. En ce qui concerne les représentations des acteurs économiques, en constante mutation, un effort continu de renforcement de l'image de Bâle-Ville devrait être effectué, de manière à identifier les atouts et les faiblesses de la ville et de vérifier les effets des mesures déjà entreprises dans ce domaine. Les acteurs cibles devraient être informés de manière régulière sur les avantages d'une localisation bâloise. Le marketing urbain pourrait réunir les différents atouts qui concourent à l'image de la ville du point de vue suisse et étranger et, sur la base d'un consensus entre les différents acteurs, des objectifs et des orientations à développer pourraient être mis en oeuvre. Un premier pas serait de rassembler l'ensemble des activités de promotion conduites dans le cadre du marketing urbain, de manière à véhiculer une image uniforme vis-à-vis de l'extérieur. Un second pas serait que la promotion économique dispose de suffisamment d'influence sur les cadres et les décisions administratives qui s'avèrent néfastes pour l'économie. Cela pourrait être envisagé dans un contexte qui autoriserait que de nouvelles lois soient évaluées en fonction de leurs effets sur l'économie et que soient évitées les mesures qui pourraient freiner le développement économique.

\section{BIBLIOGRAPHIE}

Basel Economics (éd.) (2006). - Die volkswirtschaftliche Bedeutung der Messe Schweiz, Bâle, p.1-41.

Crédit Suisse (éd.) (2004). - Wirtschaftsraum Basel. Struktur und Perspektiven, Zurich, Economy \& Policy Consulting, p.1-52.

Département de l'économie et des affaires sociales du canton de Bâle-Ville (2003). Wirtschaftsbericht 2003, Bâle.

Frey R.L. (1989). - Wirtschaftsstandort Basel: Stärken und Schwächen. Ein Anforderungsprofil für förderungswürdige Betriebe zuhanden der Basler Wirtschaftsförderung, Bâle, WWZ Studien 9, p.1-19.

Gehringer P.O., Kuhn S. (2006) - « Gewinnsteuern - Schweiz belegt Mittelfeld im weltweiten Wettbewerb », Der Schweizer Treuhänder, n¹2, p.937-944.

Hauser H., Bradke S. (1992). - EWR-Vertrag, EG Beitritt, Alleingang - Wirtschaftliche Konsequenzen für die Schweiz, Reger.

Heineberg H. (2006). - Stadtgeographie, Paderborn, Verlag Ferdinand Schöningh.

Kampschulte A., Schneider-Sliwa R. (1999). - Das Image von Basel - Steuerungsinstrument für die Stadtentwicklung? Basler Feldbuch Band 16, Bâle, p.1-125.

Odermatt A., Wachter D. (2004). - Schweiz - Eine moderne Geographie, Zurich, NZZ.

Office statistique du canton de Bâle-Ville (1999). - Bevölkerungsstruktur und Bevölkerungsdynamik beider Basel, Bâle, p.1-64. 
Office statistique du canton de Bâle-Ville (2001). - Wirtschaftsstruktur und Wirtschaftsdynamik der Nordwestschweiz. Stadt und Region 2, Bâle, p.1-81.

Office statistique du canton de Bâle-Ville (2004). - Politikplan 2005-2008 - Das Planungs- und Steuerungsinstrument des Regierungsrates, Bâle.

Office statistique du canton de Bâle-Ville (2005). - Bevölkerungsbericht 2005, Bâle, p.1-215.

Office statistique du canton de Bâle-Ville (2006). - Medienmitteilung vom 30.11.2006 - Erste Ergebnisse der Betriebszählung, Bâle, p.1-6.

Pfaff-Schlec H. (éd.) (1997). - Stadtmarketing und kommunales Audit. Chance für eine ganzheitliche Stadtentwicklung, Berlin, Heidelberg, New York, Springer.

\section{Sources internet :}

BADAC Banque de données des cantons et des villes suisses (2007): Importations et exportations 2001, en milliers de francs suisses et en \% http://www.badac.ch/FR/tableaux/cantons/ index_all.html\#eco (accédé le 24 juin 2009).

Office Fédéral de la Statistique (2007) http://www.bfs.admin.ch/bfs/portal/fr (accédé le 24 juin 2009).

Location Switzerland (2007) http://www.locationswitzerland.com (accédé le 24 juin 2009).

\section{RÉSUMÉS}

L'image d'un lieu est influencée par des facteurs réels et par des représentations. Parmi ceux-ci figurent en premier plan les milieux innovants et créatifs, qui sont à l'origine de la création d'une forte valeur ajoutée. Les entreprises de haute technologie et les laboratoires de recherche, innovants et à forte intensité de connaissance, sont considérés comme les «locomotives » du développement économique régional et national. Au moyen d'enquêtes par questionnaires, des données et des témoignages ont été recueillis relativement à l'image de la ville de Bâle, en tant que lieu d'implantation d'activités économiques et scientifiques. A partir de ces données, des recommandations peuvent être formulées en ce qui concerne la promotion économique et le marketing urbain. L'enquête permet d'évaluer dans quelle mesure les facteurs répertoriés sont valables à Bâle-Ville. En outre, le travail permet aux entreprises ou aux laboratoires de recherche d'évaluer l'importance d'un certain nombre de facteurs de localisation pour leurs activités. Les informations récoltées par l'enquête devraient fournir des indications sur l'image de Bâle auprès de ce groupe cible et permettre de déterminer à quel niveau se situent les besoins éventuels.

The image of a location depends on its real and perceived location factors. Generally, those characteristics which nurture an innovative and stimulating environment, also contribute to greater creation of value. Knowledge intensive, innovative high technology industries and research groups are considered to be the driving force of regional and national economic development. Use was made here of a written survey to collect empirical opinions and data about the image of the city of Basel as economic and science location. The aim was to formulate suggestions to support economic development and urban marketing. The survey participants were asked to rate characteristics with reference to the location of Basel. Further, they were asked to rate these same characteristics according to abundance and importance for the own company, group or institution. The results allow a picture to be made of how the target group sees the city of Basel and points to areas for possible improvement. 
Das Image eines Standorts wird durch vorhandene und wahrgenommene Standortfaktoren beeinflusst. Hierbei treten vor allem Eigenschaften in den Vordergrund, die innovative und kreative Milieus und dadurch eine hohe Wertschöpfung schaffen. Wissensintensive, innovative Hochtechnologieunternehmen und Forschungsgruppen gelten als „Zugpferde“ der regionalen wie nationalen wirtschaftlichen Entwicklung. Mittels schriftlicher Befragung wurden empirische Aussagen und Daten über das Image von Basel-Stadt als Wirtschafts- und Wissenschaftsstandort gesammelt, aus welchen sich konkrete Empfehlungen für die Wirtschaftsförderung und das Stadtmarketing ableiten lassen. Die Befragungsgruppe sollte Eigenschaften danach bewerten, in welchem Masse diese auf den Standort Basel-Stadt zutreffen. Weiter sollten Standortfaktoren in Hinblick auf das Vorhandensein in Basel-Stadt und auf deren Bedeutung für das eigene Unternehmen oder die eigene Forschungsgruppe bewertet werden. Die aus der Befragung gewonnen Aussagen sollen einen Informationen liefern, wie sich das Image von Basel-Stadt aus Sicht der Zielgruppe darstellt und wo allenfalls Handlungsbedarf besteht.

\section{INDEX}

Mots-clés : Bâle, entreprises de haute technologie, facteurs de localisation, milieu innovant

Keywords : Bâle, high technology industries, location factors, stimulating environment Schlüsselwörter : Basel, Hochtechnologieunternehmen, innovative und kreative Milieus, Standortfaktoren

\section{AUTEUR}

\section{CLAUDIA SAALFRANK}

Institute of Geography - Human Geography / Urban and Regional Studies - Department of Environmental Sciences - University of Basel - Klingelbergstrasse 27, CH 4056 Basel - Tel.: +4161-267 0727 - claudia.saalfrank@unibas.ch 